\title{
DOSIMETRIA DOS CONES RADIOCIRÚRGICOS RADIONICS DE DIÂMETROS DE 5 MM A 50 MM PARA UM FEIXE DE 6 MV DE UM ACELERADOR LINEAR MEVATRON MD DIGITAL*
}

\author{
Cleber Nogueira de Souza ${ }^{1}$, Carlos Roberto Monti ${ }^{2}$
}

Resumo Os parâmetros dosimétricos de um feixe de raios $X$ de pequeno diâmetro para um sistema de radiocirurgia comercial foram medidos em água com um detector de diodo de Si do tipo p. As razões tecido-máximo, o fator de espalhamento total e os perfis dos feixes a profundidades de 5 e $10 \mathrm{~cm}$ foram medidos para 17 feixes de diâmetros circulares de $5 \mathrm{~mm}$ a $50 \mathrm{~mm}$, em incrementos de $2,5 \mathrm{~mm}$. Os fatores de espalhamento totais caíram lentamente, de 0,947 para 0,888 entre os cones de $50 \mathrm{~mm}$ e 12,5 mm de diâmetro (variação de 7\%); para os cones entre $10 \mathrm{~mm}$ e $5 \mathrm{~mm}$ de diâmetro, esta queda foi bem maior, de 0,854 para 0,666 (variação de 28\%). Os valores obtidos para a relação tecido-máximo são consistentes com dados publicados. Os perfis dos feixes foram medidos nas direções $x$ e $y$, e estão dentro de $0,2 \mathrm{~mm}$ para todos os cones entre as duas direções. A medida da largura à meia-altura se encontra dentro de $1 \mathrm{~mm}$ com o diâmetro nominal dos cones. Unitermos: Detector de diodo. Radiocirurgia. Dosimetria de feixe de fótons.

\begin{abstract}
Dosimetry of the Radionics radiosurgery cones from $5 \mathrm{~mm}$ to $50 \mathrm{~mm}$ diameter for the $6 \mathrm{MV}$ beam of a Mevatron MD digital linac.

The dosimetric parameters of small diameter photon beams of a commercially available radiosurgery system were measured in a water phantom using a p-type Si photon diode. Tissue maximum ratios, total scattering factor and beam profiles at 5 and $10 \mathrm{~cm}$ depth were measured for 17 circular beams ranging from $5 \mathrm{~mm}$ to 50 $\mathrm{mm}$ in diameter, in $1.5 \mathrm{~mm}$ steps. The total scattering factor decreased slowly from 0.947 to 0.888 for the cones with diameter between $50 \mathrm{~mm}$ and $12.5 \mathrm{~mm}$ (7\% variation) whereas for cones with diameter between $10 \mathrm{~mm}$ and $5 \mathrm{~mm}$ the factor decreased more steeply, from 0.854 to 0.666 (28\% variation). These tissue maximum ratio data are consistent with the data published by other authors. The beam profiles measured in the $x$ and $y$ directions were aproximately $0.2 \mathrm{~mm}$ between the two directions for all cone sizes. The full widths at half maximum were within $1 \mathrm{~mm}$ of the nominal cone sizes.

Key words: Diode detector. Radiosurgery. Photon beam dosimetry.
\end{abstract}

\section{INTRODUÇÃO}

A radiocirurgia estereotáxica para o tratamento de lesões cerebrais com feixes de fótons de energia alta de aceleradores lineares é uma modalidade de tratamento complexa já bem estabelecida. No entanto, as medidas dos fatores de rendimento dos cones, razão tecido-máximo (RTM), perfis de feixes de diâmetros muito pequenos $(5-10 \mathrm{~mm})$ e de diâmetros pequenos (12,5-40 mm) ainda apresentam significativo desafio.

Conforme observado por Wu et al. ${ }^{(\mathbf{1})}$, campos de raios $\mathrm{X}$ de diâmetros pequenos apresentam perdas de equilíbrio ele-

* Trabalho realizado no Instituto do Radium de Campinas, Campinas, SP

1. Doutor, Físico do Instituto de Pesquisas Energéticas e Nucleares, São Paulo, SP.

2. Doutor, Rádio-oncologista do Instituto do Radium de Campinas.

Endereço para correspondência: Dr. Cleber Nogueira de Souza. Instituto de Pesquisas Energéticas e Nucleares. Travessa R, 400, Cidade Universitária. São Paulo, SP, 05508-900. Aceito para publicação em 5/10/2000 trônico lateral, exigindo pequenas correções da dose. A resolução espacial de medidas de perfil dos feixes e penumbras é dependente do tamanho do detector, o que é agravado pelos diferentes tipos de detectores - câmaras de ionização, diodos e dosímetros termoluminescentes, que apresentam tamanhos próximos aos tamanhos dos campos de tratamento. Assim, medidas exatas dos fatores de rendimento ou outros parâmetros relacionados - fatores de espalhamento do fantoma e fatores de espalhamento total, que são dependentes das características do emissor de radiação exigem cuidados especiais ${ }^{(2,3)}$.

Alguns métodos de dosimetria aplicáveis - filmes radiocrômicos, detectores de diamante e dosimetria por varredura com microdosímetros Fricke em gel infundida - para verificação da dose são caros e não-disponíveis na maioria dos serviços. Além disso, embora existam dados publicados para os cones de diâ- metros pequenos (acima de 12,5 mm), para os cones de diâmetros muito pequenos (5-10 mm) estes dados são restritos $^{(4-7)}$

O Instituto do Radium de Campinas adquiriu um sistema de radiocirurgia com 17 cones divergentes, com diâmetros de $5 \mathrm{~mm}$ a $50 \mathrm{~mm}$, em incrementos de $2,5 \mathrm{~mm}$, definidos a $100 \mathrm{~cm}$, para uso de um feixe de $6 \mathrm{MV}$ de um acelerador linear. Os resultados dos fatores de espalhamento totais, RTM e perfis do feixe são apresentados. Estes resultados foram comparados com dados já publicados.

\section{MATERIAIS E MÉTODOS}

\section{O sistema de radiocirurgia}

$\mathrm{O}$ sistema de radiocirurgia, marca $\mathrm{Ra}-$ dionics, do Instituto do Radium de Campinas, utiliza feixe de fótons de $6 \mathrm{MV}$ de um acelerador linear Digital Mevatron MD, Siemens. Este equipamento produz feixes de fótons de 6 e $15 \mathrm{MV}$, e 
feixes de elétrons de 6 até $14 \mathrm{MeV}$. O sistema Radionics inclui um fixador de cabeça Brown-Robert-Wells (BRW) para o sistema de radiocirurgia estereotáxica, e um fixador recolocável Gill-Thomas-Cosman (GTC) para os tratamentos fracionados.

Para se definir o sistema de coordenadas estereotáxicas, um fixador estereotáxico rígido é instalado no crânio do paciente, por intermédio da inserção de parafusos. Com este tipo de fixação, o deslocamento do isocentro pode ser minimizado $(\leq 1 \mathrm{~mm})$ entre os procedimentos de obtenção das imagens para o planejamento e para o tratamento. Uma fração única de dose de radiação pode, dessa forma, ser prescrita e liberada com a exatidão adequada. Sistemas fixadores não-invasivos são também empregados com uma reprodutibilidade menor $(\leq 3$ $\mathrm{mm})$, o que é aceitável entre aplicações ${ }^{(\mathbf{8})}$. Tais fixadores podem ser utilizados para os tratamentos fracionados. Atualmente, o fixador de cabeça GTC é um dos sistemas recolocáveis mais usados em terapia fracionada ${ }^{(9)}$.

O sistema de planejamento tridimensional Radionics, que emprega o algoritmo de dose Xknife, é usado para o planejamento do tratamento. Um equipamento de tomografia computadorizada helicoidal marca General Electric, modelo High-Speed, e um equipamento de ressonância magnética Siemens, modelo Magneton, são utilizados para a obtenção das imagens, delineamento do volume-alvo e reconstrução volumétrica.

Um sistema de localização do isocentro, consistindo de quatro luzes de laser verdes, instalado nas paredes e teto, é empregado para testar a exatidão geométrica. A exatidão do "gantry", colimadores e rotação da mesa de tratamento foram verificados durante o comissionamento do sistema radiocirúrgico. A exatidão no alinhamento e a verificação do isocentro e alvo foram realizadas conforme estabelecidos no manual do operador Radionics, por Tsai et al. ${ }^{(\mathbf{1 0})} \mathrm{e}$ pelo protocolo AAPM Report no. 54(11).

\section{Dosimetria}

Um detector de diodo de junção do tipo p, Scanditronix, foi empregado em todas as medidas. Este diodo tem volume sensível de $0,3 \mathrm{~mm}^{3}$, área superficial de $4,9 \mathrm{~mm}^{2}$ e espessura efetiva de 0,06 $\mathrm{mm}$. O ponto efetivo de medida está a uma profundidade de $0,6 \mathrm{~mm}$ da sua superfície frontal. Este detector foi acoplado a um sistema de aquisição de dados computadorizados Wellhöfer, versão WP700, e utilizado em simulador de água. Todas as medidas foram realizadas com o detector orientado na direção do feixe de radiação, onde o diodo apresenta sua menor projeção para o feixe incidente. As características de resolução espacial deste detector foram analisadas por Rustgi e Frye ${ }^{(\mathbf{1 2})}$, que concluíram que são comparáveis às mesmas medidas feitas com dosimetria fotográfica.

Os cones de radiocirurgia divergentes têm $10 \mathrm{~cm}$ de espessura e distam 24 $\mathrm{cm}$ do isocentro. A abertura dos colimadores foi mantida em $6 \times 6 \mathrm{~cm}$ para todos os diâmetros dos cones.

O sistema Xknife calcula a dose a uma profundidade $d$, em um ponto dado pela seguinte equação:

$D(c, r, d)=M \cdot S_{t}(c) \cdot \operatorname{RTM}(c, d) \cdot \operatorname{ROFA}(c, r, d)$

onde: $c$ é o tamanho do cone em milímetros; $r$ é a distância radial do eixo central do feixe; $d$ é a profundidade do ponto dentro do paciente ao longo do eixo central do feixe; $M$ é o fator que relaciona as unidades monitoras à dose (cGy) ao campo de $10 \times 10 \mathrm{~cm} \mathrm{em} d_{\max }$; $S_{t}$ é o fator de espalhamento total, que é a razão entre o rendimento em $d_{\max }$ para um tamanho de cone determinado e o rendimento em $d_{\max }$ para o campo de 10 $\times 10 \mathrm{~cm}^{(\mathbf{1 0})}$. A $R T M(c, d)$ e a razão de "offaxis" - ROFA $(c, r, d)$ - têm as definições convencionais.

Todos os parâmetros foram medidos utilizando a geometria isocêntrica (distância fonte-eixo = $100 \mathrm{~cm}$ ). A ROFA foi medida nas profundidades de $5 \mathrm{~cm}$ e $10 \mathrm{~cm}$. O rendimento da máquina em $d_{\max }$ relativo às unidades monitoras (UM) para um campo quadrado de $10 \times 10$ $\mathrm{cm}$ para uma distância foco-superfície de $100 \mathrm{~cm}$ foi de 1,00 cGy/UM em água. $M$ é calculado multiplicando-se este rendimento pelo fator do inverso do quadrado da distância $(100+1,5)^{2} /(100)^{2}=$ $1,03 \mathrm{cGy} / M$.
Para um cálculo rápido e manual das unidades monitoras, aplicado pelo programa de garantia da qualidade dos planejamentos, uma forma funcional para a RTM é(13):

$$
R T M(c, d)=A(c) \cdot e^{-\mu(c) \cdot d}
$$

onde: $A(c)$ e $\mu(c)$ são os parâmetros de ajuste dos dados medidos para um cone cujo diâmetro do campo, projetado no isocentro, é $c$. As unidades monitoras para liberar a dose prescrita DP podem ser calculadas se o peso do arco, $W$, e a profundidade média, $d_{m}$, forem conhecidos, conforme a equação seguinte, que inclui a equação 2 na sua fórmula. Em radiocirurgia, o peso do feixe é definido como a razão do ângulo do arco de um feixe para o ângulo de arco total para todos os feixes.

$$
M=\frac{D P \cdot W}{S_{t} \cdot A \cdot e^{-\mu \cdot d_{m}}}
$$

\section{RESULTADOS}

\section{Fatores de espalhamento totais, $S_{t}$}

Os fatores de espalhamento totais são apresentados na Figura 1. Esses fatores diminuíram em 7\%, de 0,947 para 0,888, entre os cones de $50 \mathrm{~mm}$ e de $12,5 \mathrm{~mm}$, o que é consistente com resultados anteriores $^{(\mathbf{1 2})}$. Entre os cones de $10 \mathrm{~mm}$ e $5 \mathrm{~mm}$, esta diminuição é bem maior, de 0,854 para 0,666 . De acordo com a Figura 1 , os valores de $S_{t}$ deste estudo são cerca de $1 \%$ maior que aqueles medidos por Rustgi e Frye ${ }^{(12)}$ utilizando o mesmo detector, isto é, um diodo Scanditronix. A razão provável disto é que se utilizou, neste estudo, o detector com sua menor projeção na direção do feixe. Em relação aos valores de Das et al. ${ }^{(14)}$, pode-se dizer que os resultados são coincidentes, dentro dos limites dos erros experimentais. Acrescente-se a isto que os fatores de espalhamento totais apresentam alguma dependência com cada máquina - Serago et al. ${ }^{(5)}$ apresentaram fatores de rendimento relativos de 0,90 para um cone de diâmetro de $10 \mathrm{~mm}$ para um feixe de 6 MV de um Varian 6/100 e de 0,74 para um cone de $8 \mathrm{~mm}$ para um feixe de $6 \mathrm{MV}$ de um Clinac 2500.

A influência da abertura dos colimadores principais sobre $S_{t}$ foi também verificada. A Tabela 1 apresenta estes re- 

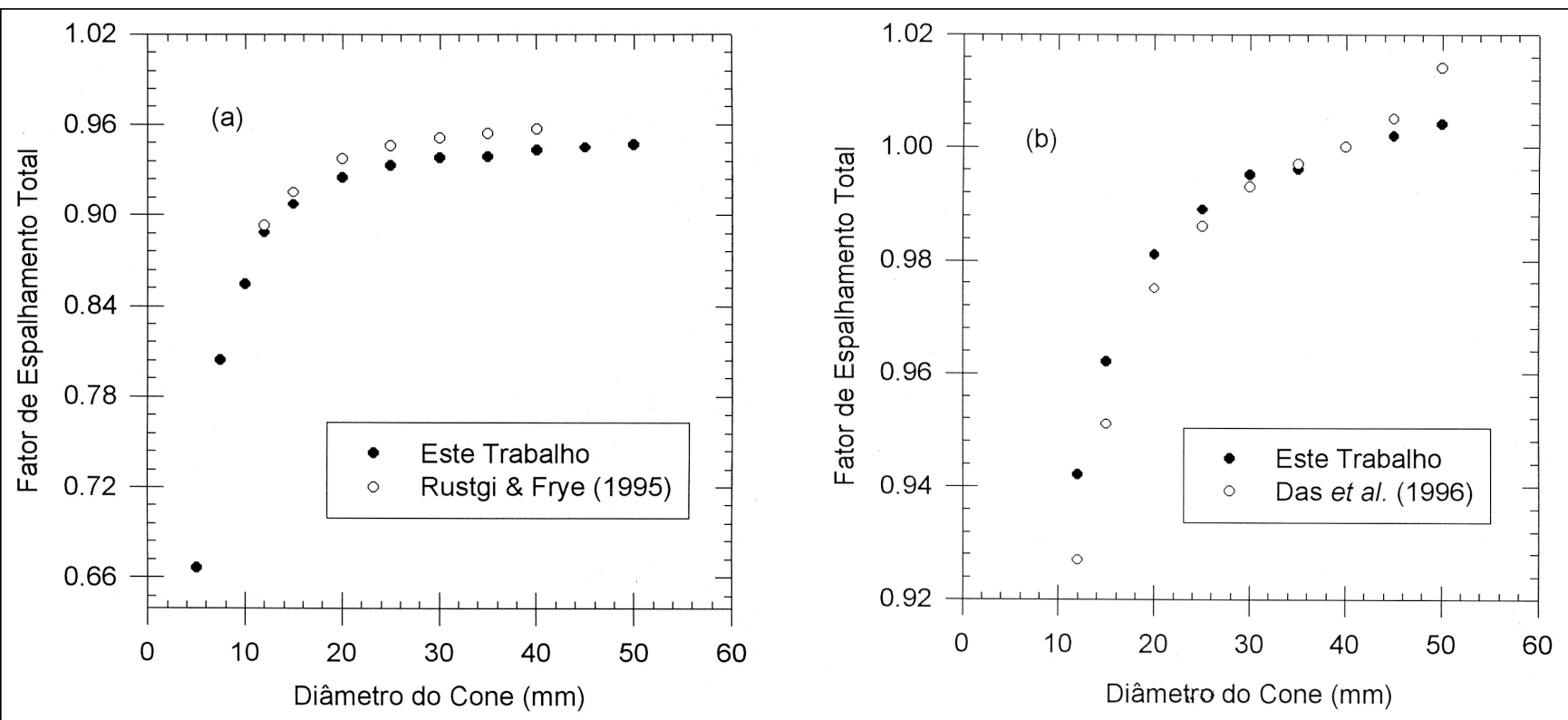

Figura 1. Fatores de espalhamento totais relativos: (a) ao campo de referência $10 \times 10 \mathrm{~cm}$; (b) ao cone de diâmetro de $40 \mathrm{~mm}$.

sultados para a abertura de uso dos colimadores durante os tratamentos radiocirúrgicos $-6 \times 6 \mathrm{~cm}-\mathrm{e}$ para a abertura dos colimadores em $10 \times 10 \mathrm{~cm}$. Os fatores de espalhamento foram sempre iguais ou maiores para a maior abertura de colimador, chegando a uma variação máxima de $2,4 \%$ para o cone maior, que é o de $50 \mathrm{~mm}$. Serago et al. ${ }^{(5)}$ encontraram variações maiores e utilizaram uma abertura de colimador de $5 \times 5 \mathrm{~cm}$, menor que a abertura de colimador utilizada neste estudo.

Tabela 1 Influência da abertura dos colimadores no rendimento relativo dos diferentes tamanhos dos cones normalizados para o campo $10 \times 10 \mathrm{~cm}^{2}$.

\begin{tabular}{|c|c|c|}
\hline Cone $(\mathrm{mm})$ & $\begin{array}{c}\text { Colimador } \\
6 \times 6 \mathrm{~cm}^{2}\end{array}$ & $\begin{array}{c}\text { Colimador } \\
10 \times 10 \mathrm{~cm}^{2}\end{array}$ \\
\hline 10 & 0,854 & 0,855 \\
20 & 0,925 & 0,943 \\
30 & 0,938 & 0,959 \\
40 & 0,943 & 0,966 \\
50 & 0,947 & 0,970 \\
\hline
\end{tabular}

\section{Razão tecido-máximo}

Os dados da RTM são apresentados na Tabela 2 para todos os cones e na Figura 2 para os cones mais representativos. A profundidade de $d_{\max }$ para os di-

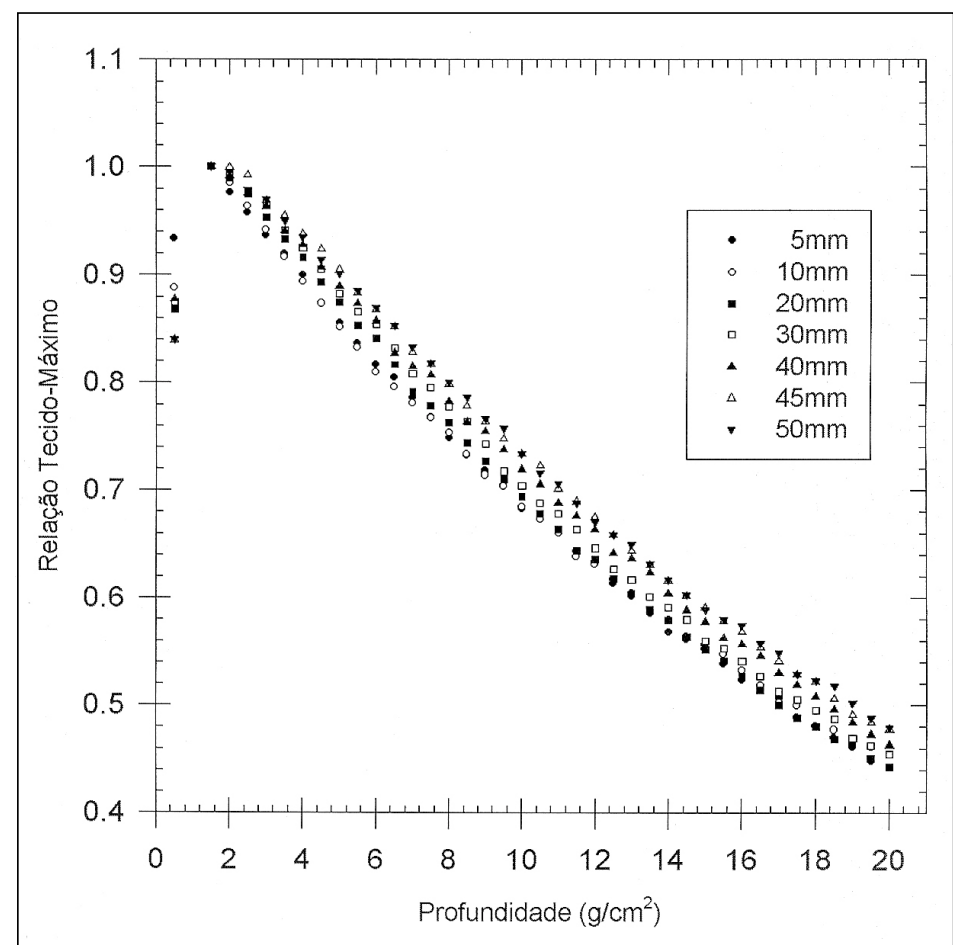

Figura 2. Valores da relação tecido-máximo do feixe de fótons de $6 \mathrm{MV}$ para alguns cones radiocirúrgicos.

versos cones apresentou variações erráticas, que podem ser explicadas por incertezas experimentais. $\mathrm{O} d_{\max }$ pode ser, dentro dos limites da exatidão experimental, considerado como constante e localizado a $1,5 \mathrm{~cm}$ de profundidade. As variações da RTM entre os campos de 5 $\mathrm{mm}$ e $50 \mathrm{~mm}$ são de $5 \%$ a $5 \mathrm{~cm}$ de profundidade, $7 \%$ a $10 \mathrm{~cm}$ de profundidade, e $9 \%$ à profundidade de $20 \mathrm{~cm}$. Na Tabela 3 é apresentada uma comparação com dados publicados ${ }^{(14)}$. Os resultados apresentados por Das et al. ${ }^{(14)}$ mostram uma coincidência geral para todos os 
Tabela 2 Relação tecido-máximo para os cones radiocirúrgicos circulares Radionics.

\begin{tabular}{|c|c|c|c|c|c|c|c|c|c|c|c|c|c|c|c|c|c|}
\hline \multirow{2}{*}{$\begin{array}{l}\text { Prof. } \\
(\mathrm{cm})\end{array}$} & \multicolumn{17}{|c|}{ Diâmetro } \\
\hline & $5 \mathrm{~mm}$ & $7,5 \mathrm{~mm}$ & $10 \mathrm{~mm}$ & $12,5 \mathrm{~mm}$ & $15 \mathrm{~mm}$ & $17,5 \mathrm{~mm}$ & $20 \mathrm{~mm}$ & $22,5 \mathrm{~mm}$ & $25 \mathrm{~mm}$ & $27,5 \mathrm{~mm}$ & $30 \mathrm{~mm}$ & $32,5 \mathrm{~mm}$ & $35 \mathrm{~mm}$ & $37,5 \mathrm{~mm}$ & $40 \mathrm{~mm}$ & $45 \mathrm{~mm}$ & $50 \mathrm{~mm}$ \\
\hline 0,0 & 0,461 & 0,453 & 0,461 & 0,453 & 0,441 & 0,429 & 0,429 & 0,430 & 0,426 & 0,429 & 0,442 & 0,427 & 0,427 & 0,426 & 0,426 & 0,407 & 0,408 \\
\hline 0,5 & 0,934 & 0,928 & 0,888 & 0,924 & 0,899 & 0,867 & 0,867 & 0,870 & 0,862 & 0,868 & 0,874 & 0,872 & 0,874 & 0,876 & 0,877 & 0,839 & 0,840 \\
\hline 1,5 & 1,000 & 1,000 & 1,000 & 1,000 & 1,000 & 1,000 & 1,000 & 1,000 & 1,000 & 1,000 & 1,000 & 1,000 & 1,000 & 1,000 & 1,000 & 1,000 & 1,000 \\
\hline 2,0 & 0,976 & 0,977 & 0,984 & 0,987 & 0,987 & 0,988 & 0,989 & 0,990 & 0,991 & 0,996 & 0,995 & 0,991 & 0,992 & 0,993 & 0,991 & 0,999 & 0,995 \\
\hline 2,5 & 0,957 & 0,958 & 0,963 & 0,965 & 0,972 & 0,968 & 0,974 & 0,976 & 0,977 & 0,974 & 0,976 & 0,975 & 0,977 & 0,980 & 0,976 & 0,992 & 0,978 \\
\hline 3,0 & 0,937 & 0,934 & 0,941 & 0,946 & 0,950 & 0,954 & 0,953 & 0,960 & 0,958 & 0,956 & 0,965 & 0,963 & 0,956 & 0,960 & 0,963 & 0,969 & 0,969 \\
\hline 3,5 & 0,920 & 0,910 & 0,917 & 0,926 & 0,929 & 0,929 & 0,933 & 0,938 & 0,941 & 0,939 & 0,941 & 0,944 & 0,939 & 0,941 & 0,941 & 0,955 & 0,950 \\
\hline 4,0 & 0,899 & 0,890 & 0,894 & 0,901 & 0,908 & 0,912 & 0,916 & 0,912 & 0,913 & 0,928 & 0,925 & 0,920 & 0,920 & 0,929 & 0,928 & 0,938 & 0,935 \\
\hline 4,5 & 0,874 & 0,868 & 0,873 & 0,888 & 0,889 & 0,886 & 0,893 & 0,894 & 0,899 & 0,906 & 0,905 & 0,905 & 0,901 & 0,907 & 0,907 & 0,924 & 0,914 \\
\hline 5,0 & 0,855 & 0,848 & 0,851 & 0,861 & 0,862 & 0,866 & 0,874 & 0,879 & 0,878 & 0,883 & 0,882 & 0,884 & 0,883 & 0,890 & 0,889 & 0,905 & 0,901 \\
\hline 5,5 & 0,836 & 0,829 & 0,832 & 0,850 & 0,843 & 0,847 & 0,852 & 0,855 & 0,856 & 0,866 & 0,865 & 0,866 & 0,871 & 0,870 & 0,873 & 0,883 & 0,886 \\
\hline 6,0 & 0,817 & 0,807 & 0,810 & 0,821 & 0,824 & 0,828 & 0,840 & 0,837 & 0,837 & 0,836 & 0,854 & 0,851 & 0,854 & 0,858 & 0,857 & 0,867 & 0,870 \\
\hline 6,5 & 0,805 & 0,789 & 0,796 & 0,803 & 0,810 & 0,812 & 0,817 & 0,818 & 0,820 & 0,825 & 0,832 & 0,835 & 0,830 & 0,833 & 0,827 & 0,851 & 0,853 \\
\hline 7,0 & 0,786 & 0,771 & 0,781 & 0,783 & 0,791 & 0,785 & 0,792 & 0,797 & 0,799 & 0,807 & 0,808 & 0,810 & 0,813 & 0,816 & 0,815 & 0,828 & 0,833 \\
\hline 7,5 & 0,768 & 0,751 & 0,767 & 0,777 & 0,767 & 0,767 & 0,779 & 0,782 & 0,780 & 0,788 & 0,795 & 0,792 & 0,796 & 0,798 & 0,807 & 0,817 & 0,818 \\
\hline 8,0 & 0,748 & 0,735 & 0,754 & 0,752 & 0,748 & 0,755 & 0,763 & 0,762 & 0,767 & 0,772 & 0,778 & 0,777 & 0,779 & 0,780 & 0,782 & 0,798 & 0,800 \\
\hline 8,5 & 0,733 & 0,712 & 0,733 & 0,738 & 0,730 & 0,739 & 0,744 & 0,742 & 0,750 & 0,753 & 0,763 & 0,761 & 0,762 & 0,767 & 0,763 & 0,778 & 0,786 \\
\hline 9,0 & 0,718 & 0,699 & 0,714 & 0,716 & 0,715 & 0,721 & 0,727 & 0,728 & 0,723 & 0,737 & 0,743 & 0,741 & 0,745 & 0,748 & 0,754 & 0,764 & 0,766 \\
\hline 9,5 & 0,704 & 0,680 & 0,703 & 0,702 & 0,698 & 0,707 & 0,710 & 0,705 & 0,727 & 0,720 & 0,718 & 0,728 & 0,731 & 0,734 & 0,737 & 0,748 & 0,757 \\
\hline 10,0 & 0,683 & 0,668 & 0,685 & 0,684 & 0,690 & 0,691 & 0,694 & 0,696 & 0,702 & 0,705 & 0,704 & 0,711 & 0,716 & 0,716 & 0,720 & 0,734 & 0,734 \\
\hline 10,5 & 0,676 & 0,650 & 0,672 & 0,670 & 0,672 & 0,680 & 0,678 & 0,686 & 0,689 & 0,687 & 0,688 & 0,694 & 0,699 & 0,700 & 0,706 & 0,723 & 0,716 \\
\hline 11,0 & 0,662 & 0,641 & 0,660 & 0,658 & 0,656 & 0,654 & 0,664 & 0,661 & 0,672 & 0,676 & 0,678 & 0,677 & 0,686 & 0,686 & 0,689 & 0,702 & 0,706 \\
\hline 11,5 & 0,643 & 0,621 & 0,639 & 0,640 & 0,633 & 0,643 & 0,644 & 0,650 & 0,657 & 0,658 & 0,663 & 0,668 & 0,670 & 0,665 & 0,677 & 0,690 & 0,689 \\
\hline 12,0 & 0,633 & 0,614 & 0,631 & 0,624 & 0,629 & 0,629 & 0,636 & 0,638 & 0,643 & 0,644 & 0,646 & 0,648 & 0,650 & 0,658 & 0,664 & 0,676 & 0,671 \\
\hline 12,5 & 0,614 & 0,594 & 0,617 & 0,610 & 0,605 & 0,618 & 0,618 & 0,614 & 0,624 & 0,625 & 0,627 & 0,634 & 0,637 & 0,642 & 0,642 & 0,659 & 0,659 \\
\hline 13,0 & 0,602 & 0,585 & 0,605 & 0,595 & 0,593 & 0,606 & 0,604 & 0,601 & 0,611 & 0,610 & 0,617 & 0,620 & 0,624 & 0,632 & 0,637 & 0,644 & 0,650 \\
\hline 13,5 & 0,585 & 0,567 & 0,587 & 0,585 & 0,587 & 0,587 & 0,589 & 0,591 & 0,603 & 0,599 & 0,601 & 0,610 & 0,612 & 0,611 & 0,624 & 0,630 & 0,632 \\
\hline 14,0 & 0,568 & 0,562 & 0,580 & 0,569 & 0,569 & 0,573 & 0,579 & 0,575 & 0,584 & 0,584 & 0,591 & 0,595 & 0,595 & 0,602 & 0,604 & 0,616 & 0,617 \\
\hline 14,5 & 0,561 & 0,549 & 0,564 & 0,554 & 0,554 & 0,560 & 0,563 & 0,568 & 0,573 & 0,576 & 0,580 & 0,581 & 0,587 & 0,587 & 0,589 & 0,602 & 0,603 \\
\hline 15,0 & 0,556 & 0,532 & 0,552 & 0,545 & 0,542 & 0,542 & 0,551 & 0,551 & 0,560 & 0,561 & 0,560 & 0,570 & 0,573 & 0,581 & 0,578 & 0,591 & 0,589 \\
\hline 15,5 & 0,538 & 0,520 & 0,547 & 0,529 & 0,530 & 0,535 & 0,541 & 0,538 & 0,547 & 0,548 & 0,553 & 0,553 & 0,556 & 0,566 & 0,563 & 0,578 & 0,580 \\
\hline 16,0 & 0,523 & 0,507 & 0,532 & 0,519 & 0,521 & 0,524 & 0,527 & 0,532 & 0,531 & 0,534 & 0,541 & 0,542 & 0,541 & 0,551 & 0,557 & 0,568 & 0,574 \\
\hline 16,5 & 0,515 & 0,497 & 0,519 & 0,502 & 0,509 & 0,511 & 0,514 & 0,515 & 0,521 & 0,528 & 0,527 & 0,531 & 0,537 & 0,536 & 0,546 & 0,553 & 0,557 \\
\hline 17,0 & 0,506 & 0,480 & 0,502 & 0,500 & 0,493 & 0,505 & 0,500 & 0,501 & 0,510 & 0,517 & 0,513 & 0,519 & 0,525 & 0,526 & 0,530 & 0,541 & 0,548 \\
\hline 17,5 & 0,489 & 0,477 & 0,499 & 0,489 & 0,488 & 0,489 & 0,488 & 0,495 & 0,497 & 0,498 & 0,506 & 0,505 & 0,516 & 0,514 & 0,519 & 0,529 & 0,529 \\
\hline 18,0 & 0,481 & 0,465 & 0,481 & 0,476 & 0,473 & 0,478 & 0,481 & 0,482 & 0,489 & 0,493 & 0,495 & 0,496 & 0,494 & 0,504 & 0,508 & 0,522 & 0,523 \\
\hline 18,5 & 0,470 & 0,460 & 0,477 & 0,460 & 0,462 & 0,472 & 0,469 & 0,478 & 0,477 & 0,482 & 0,487 & 0,488 & 0,492 & 0,494 & 0,496 & 0,506 & 0,518 \\
\hline 19,0 & 0,460 & 0,446 & 0,465 & 0,453 & 0,456 & 0,454 & 0,464 & 0,460 & 0,468 & 0,468 & 0,469 & 0,478 & 0,479 & 0,484 & 0,484 & 0,491 & 0,502 \\
\hline 19,5 & 0,448 & 0,437 & 0,461 & 0,448 & 0,445 & 0,452 & 0,451 & 0,451 & 0,457 & 0,461 & 0,462 & 0,466 & 0,467 & 0,466 & 0,473 & 0,484 & 0,489 \\
\hline 20,0 & 0,442 & 0,425 & 0,454 & 0,434 & 0,436 & 0,440 & 0,443 & 0,444 & 0,448 & 0,451 & 0,454 & 0,460 & 0,458 & 0,460 & 0,463 & 0,478 & 0,480 \\
\hline
\end{tabular}

campos e em quase todas as profundidades. A coincidência é menor nos pontos mais profundos. Isto pode ser causado pelo aumento da sensibilidade do diodo para com os fótons de energias mais baixas. Os dados da RTM medidos foram ajustados para a equação 2 . Os coe- ficientes de $A, \mu$ e $S_{t}$ da equação 3 são apresentados na Tabela 4.

\section{Perfil do feixe}

A Figura 3 apresenta um conjunto limitado de dados de perfil do feixe. Os perfis foram medidos com o detector posicionado com sua menor projeção na direção do feixe. Os valores das ROFAs foram tomados dos perfis dos feixes. Os perfis foram medidos nas direções $\mathrm{x}$ e $\mathrm{y}$ para cada cone, e estão dentro de $0,2 \mathrm{~mm}$ para todos os cones. As ROFAs foram medidas a 5 e $10 \mathrm{~cm}$ de profundidade e 
Tabela 3 Razão tecido-máximo para diferentes tamanhos de cones.

\begin{tabular}{|c|c|c|c|c|c|c|}
\hline \multirow{2}{*}{$\begin{array}{l}\text { Prof. } \\
\text { (cm) }\end{array}$} & \multicolumn{6}{|c|}{ Diâmetro (mm) } \\
\hline & 12,5 & 15,0 & 20,0 & 30,0 & 40,0 & 50,0 \\
\hline 1,5 & $1,000 \quad(1,000)$ & $1,000 \quad(1,000)$ & $1,000 \quad(1,000)$ & $1,000(1,000)$ & $1,000 \quad(1,000)$ & $1,000 \quad(1,000)$ \\
\hline 2,0 & $0,987 \quad(0,985)$ & $0,987 \quad(0,989)$ & $0,989 \quad(0,989)$ & $0,995 \quad(0,996)$ & $0,991 \quad(0,989)$ & $0,995 \quad(0,995)$ \\
\hline 4,0 & $0,901 \quad(0,895)$ & $0,908 \quad(0,904)$ & $0,916 \quad(0,909)$ & $0,925(0,928)$ & $0,928 \quad(0,929)$ & $0,935 \quad(0,937)$ \\
\hline 6,0 & $0,821 \quad(0,810)$ & $0,824 \quad(0,832)$ & $0,840 \quad(0,824)$ & $0,854 \quad(0,848)$ & $0,857 \quad(0,856)$ & $0,870 \quad(0,865)$ \\
\hline 10,0 & $0,684 \quad(0,665)$ & $0,690 \quad(0,676)$ & $0,694 \quad(0,682)$ & $0,704 \quad(0,707)$ & $0,720 \quad(0,716)$ & $0,734 \quad(0,732)$ \\
\hline 14,0 & $0,569(0,544)$ & $0,569 \quad(0,556)$ & $0,579 \quad(0,569)$ & $0,591(0,589)$ & $0,604 \quad(0,598)$ & $0,617 \quad(0,614)$ \\
\hline 20,0 & $0,434 \quad(0,407)$ & $0,436 \quad(0,417)$ & $0,443 \quad(0,432)$ & $0,454 \quad(0,451)$ & $0,463 \quad(0,459)$ & $0,480 \quad(0,466)$ \\
\hline
\end{tabular}

Obs.: Os valores entre parênteses são de Das et al! ${ }^{(14)}$.

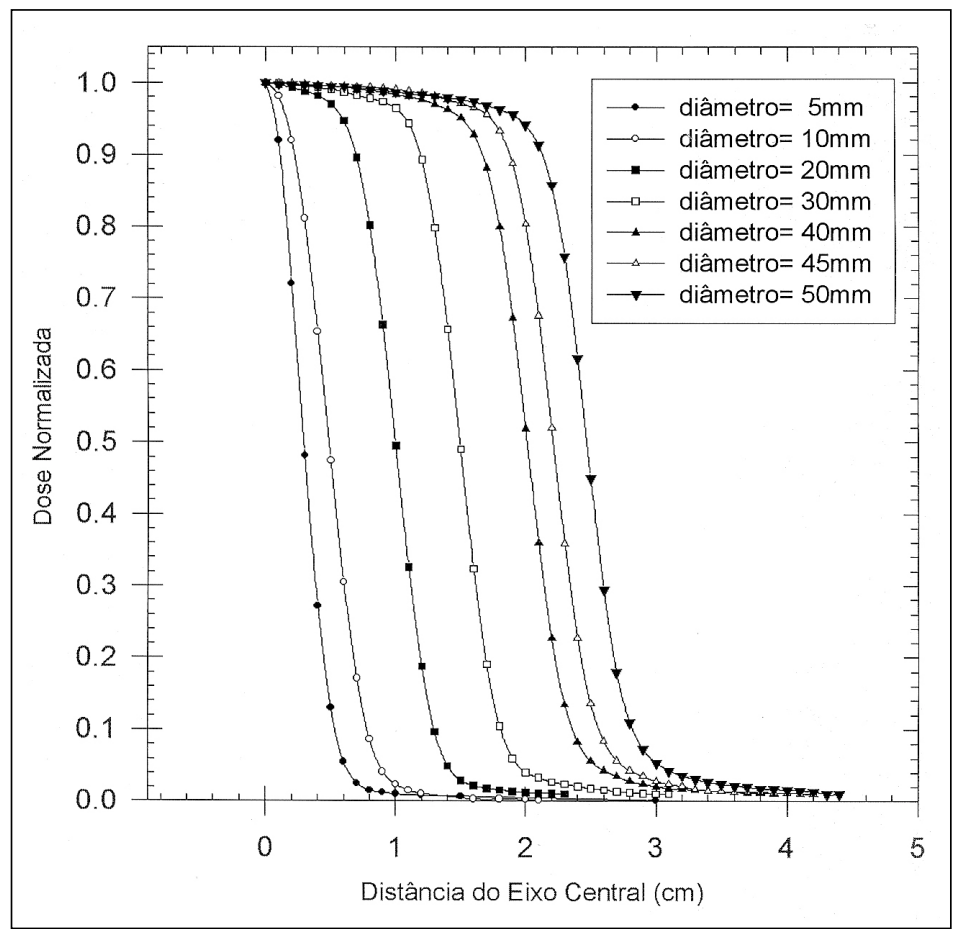

Figura 3. Perfis dos feixes de $6 \mathrm{MV}$ para alguns cones radiocirúrgicos a $5 \mathrm{~cm}$ de profundidade.

se mostraram independentes da profundidade de medida, o que já foi observado por outros autores ${ }^{(14,15)}$. A medida da largura à meia-altura se encontra dentro de $1 \mathrm{~mm}$ com o diâmetro nominal dos cones. Os dados dos perfis do feixe à profundidade de $5 \mathrm{~cm}$ foram implementados no sistema de planejamento, pois a maioria dos tumores de cabeça se encontra próximo desta profundidade.

\section{Energia do feixe}

Para o campo de $10 \times 10 \mathrm{~cm}$ a razão entre as leituras à profundidade de 20 $\mathrm{cm}$ e $10 \mathrm{~cm}$ para uma distância foco- eixo de $100 \mathrm{~cm}$ é de 0,67. Para os diversos cones, esta razão varia entre 0,63 e 0,65 . De acordo com o protocolo de dosimetria utilizado como referência ${ }^{(16)}$, isto significa uma diferença na energia ao redor de $1 \mathrm{MV}$, o que, para efeito de determinação da dose absorvida, tem pouca influência, pois a variação na razão de poder de freamento é menor que $0,5 \%$ entre os feixes de $4 \mathrm{MV}$ e $6 \mathrm{MV}^{(\mathbf{1 6})}$.

\section{CONCLUSÕES}

Os parâmetros dosimétricos do feixe de fótons de $6 \mathrm{MV}$ do acelerador linear
Tabela 4 Parâmetros para o cálculo manual ou verificação da dose, conforme a equação 3 .

\begin{tabular}{|c|c|c|c|}
\hline Diâmetro (mm) & $\mathrm{A}$ & $\mu$ & $\mathrm{S}_{\mathrm{t}}$ \\
\hline 5,0 & 1,070 & 0,0443 & 0,666 \\
7,5 & 1,070 & 0,0465 & 0,804 \\
10,0 & 1,065 & 0,0437 & 0,854 \\
12,5 & 1,083 & 0,0458 & 0,888 \\
15,0 & 1,086 & 0,0460 & 0,907 \\
17,5 & 1,085 & 0,0454 & 0,918 \\
20,0 & 1,091 & 0,0453 & 0,925 \\
22,5 & 1,092 & 0,0453 & 0,930 \\
25,0 & 1,090 & 0,0444 & 0,933 \\
27,5 & 1,094 & 0,0443 & 0,936 \\
30,0 & 1,096 & 0,0404 & 0,938 \\
32,5 & 1,093 & 0,0434 & 0,940 \\
35,0 & 1,091 & 0,0429 & 0,939 \\
37,5 & 1,093 & 0,0427 & 0,941 \\
40,0 & 1,091 & 0,0421 & 0,943 \\
45,0 & 1,101 & 0,0413 & 0,945 \\
50,0 & 1,095 & 0,0405 & 0,947 \\
\hline
\end{tabular}

Mevatron MD Digital, Siemens, foram apresentados para cones de diâmetros de $5 \mathrm{~mm}$ a $50 \mathrm{~mm}$, baseados em medidas com diodo. Estas medidas exigem cuidado na escolha do detector utilizado e variam de um equipamento para outro. No entanto, os dados aqui produzidos servirão para eventuais comparações e para orientação em medidas iniciais de feixes radiocirúrgicos.

\section{Agradecimentos}

Os autores agradecem o auxílio técnico fornecido pelo físico José Carlos Ferraz de Campos, M.Sc., da empresa Radiaton, na utilização do sistema de aquisição de dados Wellhöfer.

\section{REFERÊNCIAS}

1. Wu A, Zwicker RD, Kalend AM, Zheng Z. Comments on dose measurements for a narrow beam in radiosurgery (letter). Med Phys 1993;20:777-9.

2. Campos JCF. Dosimetria e monitoração de feixes estreitos de raios- $\mathrm{X}$, produzidos por acelerador linear de partículas, para aplicação em radiocirurgia. [Dissertação de mestrado]. Campinas: Instituto de Física, Universidade Estadual de Campinas, 1986.

3. Núñez L, Sánchez-Doblado F. Small field dosimetry in radiosurgery. Dynarad: União Européia, 1999.

4. Duggan D, Coffey C, Eisert D. Re-evaluation of physics beam parameters for small field sizes in stereoradiosurgery using a microchamber and radiochromic film (abstract). Med Phys 1995;22: 980 . 
5. Serago CF, Houdek PV, Hartmann GH, Saini DS, Serago ME, Kaydee A. Tissue maximum ratios (and other parameters) of small-circular 4, 6, 10, 15 and $24 \mathrm{MV}$ x-ray beams for radiosurgery. Phys Med Biol 1992;37:1943-56.

6. Sixel KE, Podgorsak EB. Build-up region of highenergy x-ray beams in radiosurgery. Med Phys 1993;20:761-4.

7. Westermark M, Arndt J, Nilsson B, Brahme A Comparative dosimetry in narrow high-energy photon beams. Phys Med Biol 2000;45:685-702

8. Carini S, Scielzo G, Ruggieri FG, Bistolfi F, Ravegnani M, Andreussi L. Halo ring supporting the Brown-Robert-Wells stereotactic frame for fractionated radiotherapy. Acta Neurochir (Wien) 1994;
129:92-6.

9. Gill SS, Thomas DGT, Warrington AP, Brada M. Relocatable frame for stereotactic external beam radiotherapy. Int J Radiat Oncol Biol Phys 1991; 20:599-603.

10. Tsai JS, Buck BA, Svensson GK, et al. Quality assurance in stereotactic radiosurgery using a standard linear accelerator. Int J Radiat Oncol Biol Phys 1991;21:737-48.

11. AAPM Report Number 54. Stereotactic radiosurgery. Woodbury, NY: American Institute of Physics, 1995.

12. Rustgi SN, Frye MD. Dosimetric characterization of radiosurgical beams with a diamond detector. Med Phys 1995;22:2117-21.
13. Rice RK, Hansen JL, Svensson GK, Siddon RL Measurements of dose distributions in small beams of 6 MV x-rays. Phys Med Biol 1987;32: 1087-99.

14. Das IJ, Downes MB, Corn BW, Curran WJ, Werner-Wasik M, Andrews DW. Characteristics of a dedicated linear accelerator-based stereotactic radiosurgery-radiotherapy unit. Radiother Oncol 1996;38:61-8.

15. Lutz W, Winston KR, Maleki N. A system for stereotactic radiosurgery with a linear accelerator. Int J Radiat Oncol Biol Phys 1988;14:373-81.

16. AAPM - TG-21. A protocol for the determination of absorbed dose from high-energy photon and electron beams. Med Phys 1983;10:741-71. 